PUBLIC HEALTH

\title{
Results of a randomised trial of male condom promotion among Madagascar sex workers
}

\author{
P J Feldblum, T Hatzell, K Van Damme, M Nasution, A Rasamindrakotroka, T W Grey
}

Sex Transm Infect 2005;81:166-172. doi: 10.1136/sti.2004.010074

\begin{abstract}
See end of article for authors' affiliations

Correspondence to:

Paul Feldblum, PhD, Family Health

International, PO Box 13950, Research Triangle Park, NC 27709, USA: pfeldblum@fhi.org

Accepted for publication 21 July 2004
\end{abstract}

\begin{abstract}
Objectives: To test the effect of supplementing peer promotion of male condom use with clinic based counselling, measured in terms of STI prevalence and reported male condom use.

Methods: 1000 female sex workers in Madagascar were randomised to two study arms: peer education supplemented by individual risk reduction counselling by a clinician (peer + clinic) versus condom promotion by peer educators only (peer only). STI testing was conducted at baseline and 6 months. Behavioural interviews were administered at baseline, 2, 4, and 6 months.

Results: At baseline, women in the peer only arm had prevalences of $16.0 \%, 23.6 \%$, and $12.1 \%$ for chlamydia, gonorrhoea, and trichomoniasis respectively, with an aggregate prevalence of $38.2 \%$. Baseline STI prevalences for the peer + clinic arm were slightly lower and $34.1 \%$ in aggregate. At 6 months, aggregate STI prevalence increased in the peer only arm to $41.4 \%$, whereas the aggregate prevalence diminished slightly to $32.1 \%$ in the peer + clinic arm. In logistic regression analyses, the estimated odds ratios (ORs) for chlamydia, gonorrhoea, trichomoniasis, and aggregate STI were 0.7 (95\% confidence interval 0.4 to 1.0$), 0.7(0.5$ to 1.0$), 0.8(0.6$ to 1.2$)$, and $0.7(0.5$ to 0.9$)$ respectively, comparing the peer + clinic arm with the peer only arm. The logistic regression OR for reported condom use with clients in the past 30 days increased from 1.1 at 2 months to 1.8 at 6 months, comparing the peer + clinic arm with the peer only arm, and was 1.4 overall (1.1 to 1.8). Adjustment for baseline factors changed the regression results little.

Conclusions: The impact of male condom promotion on behaviour can be heightened through more concentrated counselling on risk reduction. Persistently high STI prevalence despite increases in reported condom use by sex workers supports the need for multidimensional control programmes.
\end{abstract}

$\mathrm{P}$ romotion of male condom use is a key intervention for preventing transmission of HIV and other sexually transmitted infections (STIs), ${ }^{12}$ especially among sex workers (SWs) and others practising high risk behaviours. At the same time, obstacles to condom use are well documented, ${ }^{34}$ resistance by male partners chief among them. ${ }^{56}$ Sex workers in particular struggle to use condoms with clients. ${ }^{78}$ Reasons include limited access to condoms, the power imbalance that limits negotiation, and the lure of a higher price for unprotected sex. ${ }^{-11}$ Aversion to condom use by sex workers with their non-paying partners is equally formidable, ${ }^{12}{ }^{13}$ given the desire to distinguish between personal and professional encounters, the perceived safety of a steady partner, and the perception of mistrust implied by a request for condom use. ${ }^{1314}$

Previous research has shown that intensive, individualised educational and skill building interventions can drive up the reported consistency of male condom use, especially in high risk populations. ${ }^{815} 16$ But few studies have attempted to measure the impact of condom interventions on STI prevalence, the ultimate outcome of concern. Another poorly explored issue is the potential role of clinic based providers in condom promotion.

Madagascar serves as an appropriate setting for assessing the effect of alternative condom promotion strategies. Previous research in that country has documented inconsistent condom use within high risk populations, including sex workers, ${ }^{17}{ }^{18}$ and has revealed men's negative attitudes towards male condoms. ${ }^{19}$ Available data on sex workers in Madagascar indicate that their STI burden is high. ${ }^{21}$

We designed a randomised controlled trial (RCT) to assess the impact on behaviour and disease of adding clinic based counselling to peer education for female SWs in Madagascar.

\section{METHODS}

The study had two phases: an initial phase during which only male condoms were available, and a second phase when both male and female condoms were promoted. Analysis of the data from the second phase is still under way; we report here only on the initial male condom phase. The primary objective was to test the effect of supplementing peer promotion of male condom use with clinic based counselling, measured in terms of both STI prevalence (biological end point) and reported male condom use (behavioural end point). The study was approved by the ethics committees of the Laboratoire National de Référence (LNR) VIH/SIDA in Madagascar, and Family Health International (FHI) in North Carolina, United States.

\section{Study sites}

We worked in two public dispensaries in the capital city, Antananarivo, and in the principal port city, Tamatave. The two study cohorts have different ethnic, sociodemographic and behavioural characteristics, and the study sites were chosen to reflect diversity of Madagascar SWs and to produce findings relevant to a large proportion of urban sex workers there. We enrolled 500 female sex workers at each site.

\section{Enrolment and randomisation}

Women who completed follow up in a previous study of STI management were recruited and asked to return to the clinic for enrolment 2 months later. Half of our study participants derived from the earlier study. Other women were recruited

Abbreviations: FHI, Family Health International; LNR, Laboratoire National de Référence; RCT, randomised controlled trial; STI, sexually transmitted infections; SWs, sex workers 
by peer health educators at places where sex work occurs. All participants had to be self identified active sex workers 18 years or older with no signs of pregnancy and willing to comply with follow up visits and other study procedures. At the clinic visit, we administered oral informed consent in Malagasy. Participants signed or made their mark on the consent form in the presence of study staff and a non-study witness.

Participants were individually randomised to receive condom counselling by peer educators (hereafter referred to as the "peer only" arm), or peer counselling plus clinic based counselling ("peer + clinic" arm). A computer generated random allocation list stratified by study site was created at FHI using a block permuted approach with block sizes of 20 , 10, and four. Assignment to study arm was accomplished at the initial clinic visit after interview and examination through use of sequentially numbered sealed, opaque envelopes containing the group assignment. Clinic staff and participants were not blinded to group assignment, but STI testing and reporting were done by laboratory staff blind to group assignment.

\section{Study interventions}

All study participants received condom promotion and risk reduction counselling delivered by peer educators trained by the study. ${ }^{22}$ Counselling took place while the peer educators accompanied their assigned peer participants to scheduled bimonthly interviews at the clinics, as well as during ad hoc contacts with participants in the community. The peer educators in Tamatave were trained in social marketing and sold condoms at the lowest price available in that city. Peer educators in Antananarivo encouraged participants to purchase condoms from existing social marketing outlets. The peer educators received continuing education, supervision, and motivation at monthly meetings with the study coordinator and weekly meetings with clinic staff. The cost of these activities for the 6 month period was approximately $\$ 3660$ per site or $\$ 7.32$ per SW. The majority of this expense $(69-78 \%)$ was for monthly stipends to the peer educators. (A full cost effectiveness analysis is in preparation.)

Participants assigned to the peer + clinic arm received three bimonthly clinic counselling sessions. These were delivered in the Tamatave site by a nurse employed by the dispensary and paid a stipend by the study, and in the Antananarivo site by a physician employed by the study. These two clinicians received two week-long trainings that covered basic elements of effective counselling, including the understanding of values, identifying and assessing stages of behaviour change, $^{23}$ tailoring messages, and developing skills for effective interpersonal communication, as well as implementation of our study messages. This training was designed and led by a consultant specialising in behaviour change communication who created a programme that could be implemented and sustained in Madagascar. The counselling sessions, typically lasting approximately 15 minutes, involved a two way exchange of information on the following components: individual risk assessment; transmission and verification of basic knowledge about STIs and HIV; dual protection; demonstration of condom use with opportunity to practise using models; counselling tailored to individual circumstances; reinforcement of skills for negotiating condom use; and promotion of the "no condom = no sex" policy. Clinicians routinely referred to a counselling job aid developed for the study. Clinicians also offered a few sample condoms and advised participants to purchase additional condoms from their peer educator or another social marketing agent. The incremental cost of the clinic based reinforcement programme was approximately $\$ 765$ per site or $\$ 3.06$ per SW. Most of this expense (54-59\%) was for clinician training. The balance was additional payments to clinicians for counselling sessions.

\section{Study visits and interviews}

Participants were scheduled to make visits at 2 month intervals. At baseline and the 6 month visit, we performed clinical examinations and took specimens for STI testing. At each visit (baseline, 2, 4, and 6 months), we conducted interviews to collect data on sexual activity and reported condom use with clients (last 30 days) and non-paying partners (last act), including use of protection in most recent sex acts with clients and personal partners; condom breakage; client refusal to use condoms; reasons for condom use and non-use; and exposure to the interventions.

\section{Clinical examinations}

At each study site, one clinician examined the participants at the baseline and 6 month visits. After urine and venous blood specimen collection, the clinician performed a dermatological inspection and examination of the external genitalia. A moistened, non-lubricated speculum was used and any vaginal discharge or lesions noted. A swab sample was collected from the posterior fornix for immersion in InPouch (BioMed, San Jose, CA, USA) and a second swab from the lateral vaginal walls was rolled on a slide for Gram stain. Fluid on the speculum was tested for vaginal $\mathrm{pH}$. A bimanual examination was then done.

After the examination, all participants were treated presumptively for gonorrhoea and chlamydia with ciprofloxacin and azithromycin. Treatment was given 1 week later for syphilis (penicillin), candidiasis (nystatin), and trichomoniasis and bacterial vaginosis (metronidazole) as needed.

\section{Laboratory methods}

In Antananarivo, samples were taken directly to the LNR. Urine samples were aliquoted into two numbered cryovials and stored in designated boxes at $-20^{\circ} \mathrm{C}$. Blood specimens were screened for syphilis (MacroVue RPR Card Test, Becton Dickinson, Cockeysville, MD, USA); reactive sera were evaluated using TPPA (Fujirebio, Tokyo, Japan). The InPouch specimens were evaluated microscopically for trichomonas on the day of collection and daily during 5 days of incubation at $37^{\circ} \mathrm{C}$. The vaginal smears were Gram stained and examined microscopically for leucocytes, presence of lactobacilli, clue cells, and yeast buds and pseudohyphea.

In Tamatave, samples were taken to the adjacent laboratory, where InPouch and qualitative RPR syphilis testing was done and smears were examined. Urine and blood samples were aliquoted into cryovials and stored in designated boxes at $-20^{\circ} \mathrm{C}$ until shipped on blue ice to the LNR in Antananarivo, where RPR quality control and RPR dilutions and TPPA testing of the positive samples were done. The LNR was also responsible for quality control of microscopic analyses in Tamatave.

The frozen urine samples from both study sites were shipped on dry ice from the LNR to the University of North Carolina (Chapel Hill, NC, USA) for ligase chain reaction testing (Abbott LCx Probe System, Abbott Laboratories, Abbott Park, IL, USA) for gonococcal and chlamydial infections.

\section{Data analysis}

This study measured the impact of clinic based counselling in addition to peer condom promotion on STI prevalence at 6 months, and on the proportion of sex acts reportedly protected by condoms through 6 months. Study size calculations centred on the STI outcome. With 1000 participants, assuming $75 \%$ of participants complete this phase of the study, we would have at least $80 \%$ power to detect a $10 \%$ 


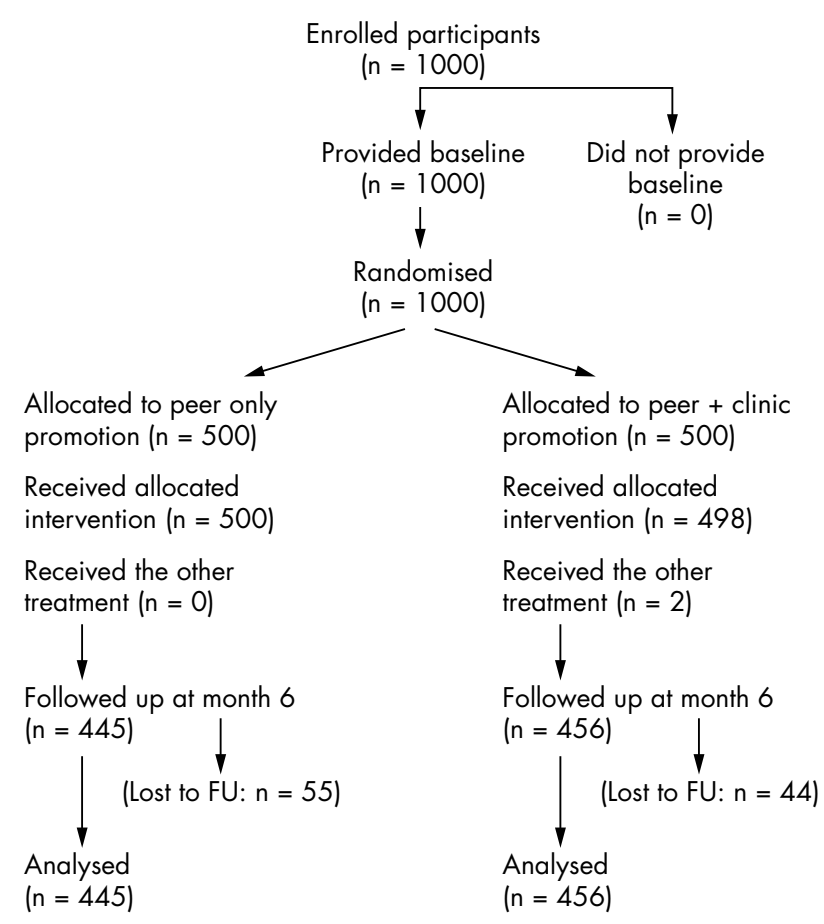

Figure 1 Participant flow chart based on STI analyses.

absolute difference in the prevalence of STI between the two groups (alpha $=0.05$, two sided test).

All analyses were performed by assigned study arm and by study site. Statistical tests were deemed significant at the $\alpha=0.05$ level. Women with missing data for a particular end point were excluded from that analysis.

For the STI analyses, we reported each STI outcome separately, and then aggregated gonococcal, chlamydial, and trichomonal infections into a dichotomous STI outcome. The main statistical analysis used logistic regression to estimate the odds ratio (OR) for prevalent STI at 6 months in the two study arms. We assumed that women with infection at baseline were treated and cured, and that the length of exposure was equal for all women. A second logistic regression analysis of STI risk adjusted for baseline covariates selected before the analysis, including prevalent STI, age, the number of partners (both clients and non-paying partners), and the number of sex acts with clients (in the past 30 days).

We calculated the reported proportion of protected sex acts with clients in the past 30 days and computed the mean value by visit, site, and study arm. For participants who had a sex act with a non-paying partner in the past 30 days, we computed the proportion whose last sex act was protected. We used logistic regression accounting for the clustering of coital acts within women and the clustering of repeated observations within women to estimate the OR for condom use at baseline, 2, 4, and 6 months in the two study arms. A second logistic regression analysis of condom use adjusted for baseline STI status, age, number of partners, and number of sex acts with clients.

\section{RESULTS}

Five hundred women were randomly assigned to peer only male condom promotion and 500 were randomly assigned to peer plus clinic based male condom counselling. Enrolment took place from February to August 2001, with follow up visits for this first phase of the study extending to June 2002; $11 \%$ and $9 \%$, respectively, of the peer arm and the peer + clinic arm never returned for follow up or did not provide specimens for STI testing and were excluded from the analysis of STI outcomes (see fig 1). Smaller numbers of other women (3\% and $4 \%$ ) missed interview visits and so were excluded from analyses of condom use (flow chart not shown).

The sociodemographic and behavioural features and physical examination results in the two study arms were quite similar at baseline (table 1). Almost half of women in Antananarivo were separated or divorced; most women in Tamatave were single with no steady boyfriend. Their median age was 26-27 years. Contraceptive use was rare. At baseline, women reported a median of five or six paying clients in the past seven days (not shown). Women in Antananarivo reported that they met virtually all their clients on the street, while in Tamatave many women met clients in bars as well as on the street (not shown).

Approximately $30 \%$ of all women had reactive RPR results at baseline, virtually all of which were confirmed positive by TPPA testing (not shown). At baseline, women in the peer

Table 1 Marital status, age, and contraceptive use at baseline by intervention group and study site

\begin{tabular}{|c|c|c|c|c|c|c|c|c|c|c|c|c|}
\hline & \multicolumn{4}{|c|}{ Antananarivo } & \multicolumn{4}{|c|}{ Tamatave } & \multicolumn{4}{|c|}{ Both sites } \\
\hline & \multicolumn{2}{|c|}{$\begin{array}{r}\text { Peer only } \\
(n=250)\end{array}$} & \multicolumn{2}{|c|}{$\begin{array}{l}\text { Peer + clinic } \\
(n=250)\end{array}$} & \multicolumn{2}{|c|}{$\begin{array}{l}\text { Peer only } \\
(n=250)\end{array}$} & \multicolumn{2}{|c|}{$\begin{array}{l}\text { Peer + clinic } \\
(n=250)\end{array}$} & \multicolumn{2}{|c|}{$\begin{array}{l}\text { Peer only } \\
(n=500)\end{array}$} & \multicolumn{2}{|c|}{$\begin{array}{l}\text { Peer + clinic } \\
(n=500)\end{array}$} \\
\hline & No & $\%$ & No & $\%$ & No & $\%$ & No & $\%$ & No & $\%$ & No & $\%$ \\
\hline \multicolumn{13}{|l|}{ Marital status* } \\
\hline Single without steady boyfriend & 27 & 10.8 & 32 & 12.8 & 151 & 60.4 & 161 & 64.4 & 178 & 35.6 & 193 & 38.6 \\
\hline Single with steady boyfriend & 53 & 21.2 & 48 & 19.2 & 92 & 36.8 & 80 & 32.0 & 145 & 29.0 & 128 & 25.6 \\
\hline Cohabiting & 57 & 22.8 & 46 & 18.4 & 0 & 0.0 & 2 & 0.8 & 57 & 11.4 & 48 & 9.6 \\
\hline Married, living with spouse & 2 & 0.8 & 4 & 1.6 & 2 & 0.8 & 3 & 1.2 & 4 & 0.8 & 7 & 1.4 \\
\hline Married, living apart from spouse & 2 & 0.8 & 2 & 0.8 & 1 & 0.4 & 2 & 0.8 & 3 & 0.6 & 4 & 0.8 \\
\hline Widowed & 11 & 4.4 & 23 & 9.2 & 1 & 0.4 & 0 & 0.0 & 12 & 2.4 & 23 & 4.6 \\
\hline Separated/divorced & 111 & 44.4 & 108 & 43.2 & 6 & 2.4 & 6 & 2.4 & 117 & 23.4 & 114 & 22.8 \\
\hline \multicolumn{13}{|l|}{ Contraceptive method use $†$} \\
\hline Pills & 15 & 6.0 & 12 & 4.8 & 18 & 7.2 & 17 & 6.8 & 33 & 6.6 & 29 & 5.8 \\
\hline Injectable & 24 & 9.6 & 31 & 12.4 & 14 & 5.6 & 24 & 9.6 & 38 & 7.6 & 55 & 11.0 \\
\hline Male condoms & 1 & 0.4 & 0 & 0.0 & 2 & 0.8 & 3 & 1.2 & 3 & 0.6 & 3 & 0.6 \\
\hline Other & 1 & 0.4 & 6 & 2.4 & 2 & 0.8 & 1 & 0.4 & 3 & 0.6 & 7 & 1.4 \\
\hline \multicolumn{13}{|l|}{ Age } \\
\hline Mean & 28.2 & & 28.5 & & 27.6 & & 28.7 & & 27.9 & & 28.6 & \\
\hline Median & 27 & & 27 & & 26 & & 28 & & 26 & & 27 & \\
\hline
\end{tabular}


Table 2 STI prevalence at baseline and 6 month follow up by intervention group and study site

\begin{tabular}{|c|c|c|c|c|c|c|c|c|c|c|c|}
\hline \multirow[b]{3}{*}{ Site } & \multirow[b]{3}{*}{ Group } & \multirow[b]{3}{*}{ Month } & \multirow{3}{*}{$\begin{array}{l}\text { No of } \\
\text { women }\end{array}$} & \multicolumn{8}{|c|}{ Type of STI } \\
\hline & & & & \multicolumn{2}{|c|}{ Chlamydia } & \multicolumn{2}{|c|}{ Gonorrhoea } & \multicolumn{2}{|c|}{ Trichomonas } & \multicolumn{2}{|c|}{ Aggregated* } \\
\hline & & & & No & $\% \dagger$ & No & $\% \dagger$ & No & $\% \dagger$ & No & $\% \dagger$ \\
\hline \multirow{4}{*}{ Antananarivo } & Peer only & 0 & 231 & 41 & 17.7 & 54 & 23.4 & 32 & 13.9 & 91 & 39.4 \\
\hline & & 6 & 231 & 52 & 22.7 & 45 & 19.7 & 52 & 22.5 & 106 & 46.3 \\
\hline & Peer + clinic & 0 & 232 & 33 & 14.3 & 45 & 19.5 & 30 & 12.9 & 83 & 35.9 \\
\hline & & 6 & 232 & 28 & 12.2 & 33 & 14.3 & 42 & 18.2 & 75 & 32.6 \\
\hline \multirow[t]{4}{*}{ Tamatave } & Peer only & 0 & 214 & 30 & 14.1 & 51 & 23.9 & 22 & 10.3 & 79 & 36.9 \\
\hline & & 6 & 214 & 21 & 10.1 & 46 & 22.3 & 27 & 12.6 & 75 & 36.1 \\
\hline & Peer + clinic & 0 & 224 & 27 & 12.1 & 45 & 20.2 & 22 & 9.8 & 72 & 32.3 \\
\hline & & 6 & 224 & 24 & 11.0 & 40 & 18.3 & 27 & 12.1 & 69 & 31.7 \\
\hline \multirow[t]{4}{*}{ Both sites } & Peer only & 0 & 445 & 71 & 16.0 & 105 & 23.6 & 54 & 12.1 & 170 & 38.2 \\
\hline & & 6 & 445 & 73 & 16.7 & 91 & 20.9 & 79 & 17.8 & 181 & 41.4 \\
\hline & Peer + clinic & 0 & 456 & 60 & 13.2 & 90 & 19.8 & 52 & 11.4 & 155 & 34.1 \\
\hline & & 6 & 456 & 52 & 11.6 & 73 & 16.3 & 69 & 15.2 & 144 & 32.1 \\
\hline
\end{tabular}

*Aggregated outcome is defined as a positive result if any of STI outcomes is positive; a negative result if all STI outcomes are negative; a missing result if any STI outcome is missing or negative or not done and none is positive.

†Percentages are calculated based on the number of women with data for the corresponding STI outcome.

only arm (both sites) had prevalences of $16.0 \%, 23.6 \%$, and $12.1 \%$ for chlamydia, gonorrhoea, and trichomoniasis respectively (table 2), with an aggregate prevalence of $38.2 \%$. The corresponding baseline STI prevalences for the peer + clinic arm were slightly lower and $34.1 \%$ in aggregate. At 6 months, the aggregate STI prevalence increased in the peer only arm to $41.4 \%$, mostly because of increases at the Antananarivo site, whereas the aggregate prevalence diminished slightly to $32.1 \%$ in the peer + clinic arm. Intervening (non-study related) treatment was reported by about $9 \%$ of women in each study arm. Generally, chlamydia and gonorrhoea prevalences decreased slightly from baseline to 6 months in both study arms at both study sites. Trichomoniasis prevalence increased from baseline to 6 months regardless of study arm or study site.

In both study arms, the self reported number of coital acts with clients in the past month remained fairly stable from baseline through 2, 4, and 6 month follow up interviews. Mean and median numbers of coital acts unprotected by male condoms decreased sharply in both study arms (not shown). Correspondingly, the mean proportions of coital acts protected by condoms increased approximately $20 \%$ in both study arms (table 3 and fig 2), while the median proportions protected by condoms increased even more. At 6 months, more than half the women in the peer + clinic arm reported $100 \%$ condom use with clients in the preceding 30 days.

About half of the women reported a sex act with a nonpaying partner in the past 30 days (table 4), more in Antananarivo than Tamatave. The proportion of women

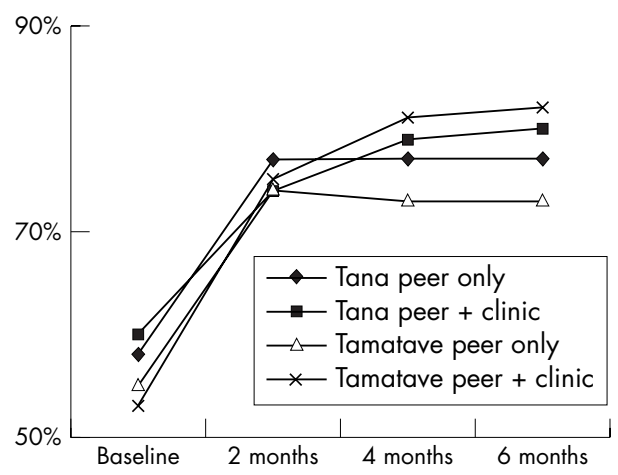

Figure 2 Mean proportion of protected sex acts with clients in the past 30 days at baseline and 2, 4, and 6 months follow up. (Tana, Antananarivo.) who reported that this last intercourse with a non-paying partner was protected by a condom increased more and was more sustained through follow up in the peer + clinic arm, but remained low.

Logistic regression analyses of STI outcomes revealed that women in the peer + clinic arm had a lower risk of individual and aggregate STI at 6 months than women in the peer only arm (table 5). The sole exception was for chlamydia at the Tamatave site, where the rate diminished more in the peer arm (an example of interaction seen in the percentages in table 2). Accounting for study site, the estimated odds ratios for chlamydia, gonorrhoea, trichomoniasis and aggregate STI were 0.7 (95\% confidence interval (CI) 0.4 to 1.0$), 0.7$ (0.5 to 1.0 ), 0.8 (0.6 to 1.2 ), and 0.7 (0.5 to 0.9 ) respectively, comparing the peer + clinic arm to the peer only arm (table 5). These results were virtually identical after adjusting for baseline covariates (not shown).

In parallel to the STI results, logistic regression analyses of reported condom use revealed that women in the peer + clinic arm used condoms more consistently, both with clients and with non-paying partners (table 6). In a model with the covariates study arm, site and time, the OR for condom use with clients increased from 1.1 at 2 months to 1.4 at 4 months to 1.8 at 6 months, and was 1.4 overall (95\% CI 1.1 to 1.8 ; table 6A). The same model produced an OR of 1.3 for condom use during the last coital act with a non-paying partner ( 1.0 to 1.8 ; table $6 \mathrm{~B}$ ), although the OR was not statistically significant and did not change over time. Adjusting for additional baseline covariates changed these figures little (not shown).

\section{DISCUSSION}

This study weakly supports the hypothesis that the impact of male condom programmes for sex workers can be heightened through more concentrated counselling on risk reduction. The estimated risk of the individual infections and of the aggregate STI outcome was reduced by $20-30 \%$ among women who received clinic based condom counselling as a supplement to peer education. The $95 \%$ confidence interval excluded 1.0 for the aggregated STI outcome.

Focusing on the absolute rather than the relative reductions in STIs suggests less salutary findings, however. Our study size calculations incorporated a $10 \%$ reduction in STI prevalence in the peer + clinic arm. The 6 month prevalences in the study arms almost reached this $10 \%$ reduction, but mainly on account of a net increase in the aggregate STI rate in the peer only arm. (We are unable to explain this counterintuitive finding, since study participants were 
Table 3 Protected coital acts with clients in the past 30 days at baseline and 2, 4, and 6 months follow up by intervention group and study site

\begin{tabular}{|c|c|c|c|c|c|c|}
\hline \multirow[b]{2}{*}{ Site } & \multirow[b]{2}{*}{ Group } & \multirow[b]{2}{*}{ Month } & \multirow{2}{*}{$\begin{array}{l}\text { No of women } \\
\text { reporting }\end{array}$} & \multicolumn{2}{|c|}{ No of women with coital act } & \multirow{2}{*}{$\begin{array}{l}\text { Mean \% of coital acts } \\
\text { protected by male condom } \\
\%^{*}\end{array}$} \\
\hline & & & & No & $\%$ & \\
\hline \multirow[t]{8}{*}{ Antananarivo } & Peer only & 0 & 242 & 238 & 98.3 & 58.0 \\
\hline & & 2 & 241 & 240 & 99.6 & 76.8 \\
\hline & & 4 & 238 & 237 & 99.6 & 76.8 \\
\hline & & 6 & 231 & 223 & 96.5 & 76.6 \\
\hline & Peer + clinic & 0 & 248 & 245 & 98.8 & 59.8 \\
\hline & & 2 & 248 & 247 & 99.6 & 74.1 \\
\hline & & 4 & 242 & 241 & 99.6 & 79.4 \\
\hline & & 6 & 232 & 226 & 97.4 & 80.3 \\
\hline \multirow[t]{8}{*}{ Tamatave } & Peer only & 0 & 245 & 244 & 99.6 & 55.1 \\
\hline & & 2 & 245 & 243 & 99.2 & 74.0 \\
\hline & & 4 & 233 & 228 & 97.6 & 73.0 \\
\hline & & 6 & 214 & 212 & 99.1 & 73.4 \\
\hline & Peer + clinic & 0 & 238 & 236 & 99.2 & 53.0 \\
\hline & & 2 & 237 & 233 & 98.3 & 75.1 \\
\hline & & 4 & 235 & 235 & 100 & 81.7 \\
\hline & & 6 & 224 & 221 & 98.7 & 82.0 \\
\hline \multirow{8}{*}{ Both sites } & Peer only & 0 & 487 & 482 & 99.0 & 56.5 \\
\hline & & 2 & 486 & 483 & 99.4 & 75.4 \\
\hline & & 4 & 471 & 465 & 98.7 & 74.9 \\
\hline & & 6 & 445 & 435 & 97.8 & 75.1 \\
\hline & Peer + clinic & 0 & 486 & 481 & 99.0 & 56.5 \\
\hline & & 2 & 485 & 480 & 99.0 & 74.6 \\
\hline & & 4 & 477 & 476 & 99.8 & 80.5 \\
\hline & & 6 & 456 & 447 & 98.0 & 81.1 \\
\hline
\end{tabular}

treated at baseline with effective single dose antibiotics for gonorrhoea, chlamydia, and trichomoniasis.) The net decrease in aggregate STI prevalence of about $2 \%$ in the peer + clinic study arm cannot be termed an effective public health intervention.

Our disappointing STI findings emerged despite marked increases in self reported male condom use. While reported condom use increased substantially in both study arms, the odds of condom use with clients and during the last coital act with a non-paying partner were $30-40 \%$ higher in the study arm benefiting from clinic based counselling, the same arm that showed greater reductions in STI prevalence. These results bolster other studies ${ }^{151624}$ indicating that repeated, individualised counselling of sex workers by health personnel can produce substantial increases in reported condom use. Our findings also suggest that individualised counselling by clinicians may further increase sex workers' condom use with non-paying partners, an outcome not achieved through other interventions. ${ }^{912} 13$ The proportion of protected acts with nonpaying partners remained fairly low, however.

Table 4 Last coital act with non-paying partners in the past 30 days at baseline and 2, 4, and 6 months follow up by intervention group and study site

\begin{tabular}{|c|c|c|c|c|c|c|}
\hline \multirow[b]{2}{*}{ Site } & \multirow[b]{2}{*}{ Group } & \multirow[b]{2}{*}{ Month } & \multirow{2}{*}{$\begin{array}{l}\text { No of women } \\
\text { reporting }\end{array}$} & \multicolumn{2}{|c|}{ No of women with coital act } & \multirow{2}{*}{$\begin{array}{l}\% \text { of women with coital act } \\
\text { protected by male condom } \\
\%^{*}\end{array}$} \\
\hline & & & & No & $\%$ & \\
\hline \multirow[t]{8}{*}{ Antananarivo } & Peer only & 0 & 242 & 155 & 64.0 & 21.3 \\
\hline & & 2 & 241 & 171 & 71.0 & 27.5 \\
\hline & & 4 & 238 & 172 & 72.3 & 25.0 \\
\hline & & 6 & 231 & 168 & 72.7 & 23.2 \\
\hline & Peer + clinic & 0 & 248 & 143 & 57.7 & 23.1 \\
\hline & & 2 & 248 & 166 & 66.9 & 32.5 \\
\hline & & 4 & 242 & 163 & 67.4 & 36.8 \\
\hline & & 6 & 232 & 176 & 75.9 & 26.7 \\
\hline \multirow[t]{8}{*}{ Tamatave } & Peer only & 0 & 245 & 87 & 35.5 & 14.9 \\
\hline & & 2 & 245 & 97 & 39.6 & 34.0 \\
\hline & & 4 & 233 & 82 & 35.2 & 18.3 \\
\hline & & 6 & 214 & 96 & 44.9 & 19.8 \\
\hline & Peer + clinic & 0 & 238 & 74 & 31.1 & 14.9 \\
\hline & & 2 & 237 & 77 & 32.5 & 32.5 \\
\hline & & 4 & 235 & 73 & 31.1 & 21.9 \\
\hline & & 6 & 224 & 82 & 36.6 & 28.0 \\
\hline \multirow[t]{8}{*}{ Both sites } & Peer only & 0 & 487 & 242 & 49.7 & 19.0 \\
\hline & & 2 & 486 & 268 & 55.1 & 29.9 \\
\hline & & 4 & 471 & 254 & 53.9 & 22.8 \\
\hline & & 6 & 445 & 264 & 59.3 & 22.0 \\
\hline & Peer + clinic & 0 & 486 & 217 & 44.7 & 20.3 \\
\hline & & 2 & 485 & 243 & 50.1 & 32.5 \\
\hline & & 4 & 477 & 236 & 49.5 & 32.2 \\
\hline & & 6 & 456 & 258 & 56.6 & 27.1 \\
\hline
\end{tabular}


Table 5 Odds ratio (OR) estimate of intervention effects (peer + clinic $v$ peer only) on STI prevalence

\begin{tabular}{llll}
\hline STI outcome and intervention effect & OR estimate* & $95 \% \mathrm{Cl}$ & p Value \\
\hline $\begin{array}{l}\text { Chlamydia } \\
\text { Overall }\end{array}$ & 0.7 & 0.4 to 1.0 & 0.03 \\
$\begin{array}{l}\text { At Antananarivo } \\
\text { At Tamatave }\end{array}$ & 0.5 & 0.3 to 0.8 & 0.00 \\
$\begin{array}{l}\text { Gonorrhoea } \\
\text { Overall }\end{array}$ & 1.1 & 0.6 to 2.0 & 0.78 \\
$\begin{array}{l}\text { Trichomoniasis } \\
\text { Overall }\end{array}$ & 0.7 & 0.5 to 1.0 & 0.08 \\
$\begin{array}{l}\text { Aggregated outcome } \\
\text { Overall }\end{array}$ & 0.8 & 0.6 tol.2 & 0.31 \\
\hline $\begin{array}{l}\text { *OR estimates were calculated from the primary analysis models. Overall OR estimates were produced from } \\
\text { models accounting for intervention and site effects only. OR estimates at each site were produced from a model } \\
\text { accounting for intervention and site effects as well as the interaction effect of the two factors. The estimates at each } \\
\text { site are presented only if there was a significant interaction effect of intervention and site. }\end{array}$ & 0.00 \\
\hline
\end{tabular}

The enormous STI burden in these Madagascar sex workers has been reported by others. ${ }^{25}$ Even with our baseline presumptive treatment with highly efficacious antibiotics, we found a considerable STI prevalence at 6 months, irrespective of study arm. The results underscore the "unforgiving" nature of highly infectious STIs: condom use needs to be quite consistent to prevent transmission. The nature of commercial sex in the poorest settings with high numbers of partners, high STI prevalence in the clients, limited diagnostic capabilities, little bargaining power among women for condom use, and strong financial incentives for non-use of protection-suggests that sex workers are likely to remain highly vulnerable to STI transmission. Multidimensional control programmes that include outreach to men and perhaps periodic presumptive treatment (PPT) of sex workers ${ }^{26}$ seem rational in this context, although the best means of applying PPT is not clear. ${ }^{27}$

This study had several limitations. Chief among them is the inaccuracy of self reports of condom use and other sexual behaviour as a result of faulty recall, social desirability bias, and poor comprehension of questions. ${ }^{28}$ We believe that the trends in self reported coital and condom data were broadly accurate in our study, while the proportions were probably exaggerated-the 6 month STI prevalences among women reporting consistent condom use with clients and non-paying partners at all three follow up interviews were somewhat lower than among other women but were still considerable. It is also worth noting that the interviewers were not blinded to the group assignment of the participants, so that social desirability bias could have differed in the study arms.

Another limitation is the long interval between STI tests that could allow resolution of some infections, and might introduce faulty recall of treatments received for symptoms experienced months before. The long reference period for intercourse with clients might have led to greater reporting inaccuracy, and the differing reference periods for intercourse with paying and non-paying partners made it difficult to assess their relative contributions to STI risk.

The fact that half of our study participants had been tested and interviewed in a previous study of STI management could have affected our results, but the proportions of women deriving from that earlier study were equal in our study arms, and that study involved less intensive counselling and less access to condoms.

The randomised comparison of the more versus less intense counselling approaches is the central strength of the current study. Other strengths include intensive monitoring of the interventions and quality control of the counselling and laboratory procedures, use of effective single dose therapies, use of highly sensitive STI tests, and high follow up rates.

Furthermore, collecting both behavioural and biological outcomes is clearly advantageous for measuring the impact of behaviour change interventions. This study echoes previous reports that healthy behaviour changes do not necessarily lead to a detectable reduction in STI rates. ${ }^{29}$ Despite marked increases in overall protection levels, and absolute decreases in the number unprotected acts with clients, opportunities for STI transmission recurred; persistently low male condom use with non-paying partners and a presumably high STI prevalence among Madagascar men were undoubtedly important. Condom use data thus served as a useful complement to the STI data by illuminating the successes and shortcomings of the behavioural intervention.

Intensive, individualised health education interventions can produce reductions in sexual risk behaviours, ${ }^{30-32}$ and, in some cases, decreases in STI prevalence. ${ }^{33} 34$ We aimed to provide the Ministry of Health, the national AIDS control

Table 6 Odds ratio (OR) estimate of intervention effect (peer + clinic $v$ peer only) on male condom use

\begin{tabular}{lccc}
\hline Intervention effect & OR estimate & $95 \% \mathrm{Cl}$ & p Value \\
\hline (A) With clients in past 30 days & $1.4^{*}$ & 1.1 to 1.8 & 0.01 \\
Overall & $1.1^{*}$ & 0.8 to 1.5 & 0.49 \\
At 2 months & $1.4^{*}$ & 1.1 to 1.8 & 0.01 \\
At 4 months & $1.8^{*}$ & 1.3 to 2.5 & 0.00 \\
At 6 months & & \\
(B) In last coital act with non-paying partner & $1.3 \dagger$ & 1.0 to 1.8 & 0.07 \\
Overall & 1.8 & \\
\hline
\end{tabular}

*OR estimates were calculated from the primary analysis models. Overall OR estimate was produced from a model accounting for the intervention, site and time effects only. OR estimates at each time were produced from a model with a significant interaction effect of intervention and time.

†OR estimates were calculated from the primary analysis model accounting for the study intervention, site and time effects only. 
programme and donors with evidence about the effectiveness and costs of alternative condom promotion strategies targeting SWs. Before this study, non-governmental organisations supported SW peer education programmes in the two study sites, but condom promotion was generally passive owing to inadequate resources. The clinic based counselling intervention tested in this study is noteworthy for its comparative simplicity, delivered in a manner that theoretically could be replicated in similar resource poor settings with functioning peer education programmes. With a recurring annual cost of approximately $\$ 2.60$ per $\mathrm{SW}$, this intervention may be worth the resources required, as part of a package of STI control services ${ }^{35}$ capitalising on health personnel already in place. The second phase of our trial will provide evidence on whether female condom distribution produces further increases in the proportion of protected sex acts, and more meaningful reductions in STI prevalence.

\section{ACKNOWLEDGEMENTS}

Partial support for this work was provided by Family Health International (FHI) with funds from the US Agency for International Development (USAID). The views expressed in this article, however, do not necessarily reflect those of USAID. FHI is an international nonprofit organisation that conducts research and provides technical assistance in health, family planning, STIs, and AIDS. The authors thank the two study teams and the Laboratoire National de Référence VIH/SIDA, for their untiring efforts in the face of civil unrest. Martina Clark helped design the self risk assessment and stages of change counselling programme. FHI economist Rick Homan completed cost analyses. We also thank Frieda Behets for extensive technical support and continuing advice throughout the study, and Rob Krysiak, Jane Parker, and Marcia Hobbs for laboratory support in the US FHI also extends thanks to Pfizer, Inc, for its generous donation of azithromycin used for treatment of study participants.

\section{CONTRIBUTORS}

The study was designed by PJF, TH, KVD, and AR; data management and analyses were done by MN and TWG; PJF and TH wrote the manuscript; all authors reviewed and take responsibility for the paper.

\section{Authors' affiliations}

P J Feldblum, T Hatzell, M Nasution, T W Grey, Family Health International, NC, USA

K Van Damme, Family Health International, Madagascar

A Rasamindrakotroka, Ministry of Health, Madagascar

Conflict of interest: None declared.

\section{REFERENCES}

1 National Institute of Mental Health (NIMH) Multisite HIV Prevention Trial Group. The NIMH Multisite HIV Prevention Trial: Reducing HIV sexual risk behavior. Science 1998;280:1889-94.

2 CDC AIDS Community Demonstration Projects Research Group. Communitylevel HIV intervention in 5 cities: final outcome data from demonstration projects. Am J Public Health 1999;89:336-45.

3 Mehryar A. Condoms: awareness, attitudes and use. In: Cleland J, Ferry B, eds. Sexual behaviour and AIDS in the developing world. Bristol, PA: Taylor and Francis, 1995.

4 Mbilinyi M, Kaihula N. Sinners and outsiders: the drama of AIDS in Rungwe. In: Baylies C, Bujra J, eds. AIDS, sexuality and gender in Africa. New York: Routledge, 2000.

5 Nuwaha F, Faxelid E, Hojer B. Predictors of condom use among patients with sexually transmitted diseases in Uganda. Sex Transm Dis 1999;26:491-5.

6 Polacsek M, Celentano D, O'Campo P, et al. Correlates of condom use stage of change: implications for interventions. AIDS Educ Prev 1999:11:38-52.

7 Estebanez P, Fitch K, Najera R. HIV and female sex workers. Bull World Health Organ 1993;71:397-412.

8 Leonard L, Ndiaye I, Kipadia A, et al. HIV prevention among male clients of female sex workers in Kaolak, Senegal: results of a peer education program. AIDS Educ Prevent 2000;12:21-37.
9 Varga C. The condom conundrum: barriers to condom use among commercial sex workers in Durban, South Africa. Afr J Reprod Health 1997; 1:74-88.

10 Wojicki JM, Malala J. Condom use, power and HIV/AIDS risk: sex-workers bargain for survival in Hillbrow/Joubert Park/Berea, Johannesburg. Soc Sci Med 2001;53:99-121.

11 Karim QA, Karim SS, Soldan K, et al. Reducing the risk of HIV infection among South African sex workers: socioeconomic and gender barriers. Am J Public Health 1995:85:1521-5.

12 Egger M, Pauw J, Lopatatzidis A, et al. Promotion of condom use in a high-risk setting in Nicaragua: a randomized controlled trial. Lancet 2000;355:2101-5.

13 Walden VM, Mwangulube K, Makhumula-Nkhoma P. Measuring the impact of a behaviour change intervention for commercial sex workers and their potential clients in Malawi. Health Educ Res 1999;14:545-54.

14 Voeten HA, Egesah OB, Onidege MY, et al. Clients of female sex workers in Nyanza province, Kenya: a core group in STD/HIV transmission. Sex Transm Dis 2002;29:444-52.

15 Ngugi EN, Wilson D, Sebstad J, et al. Focused peer-mediated educational programs among female sex workers to reduce sexually transmitted disease and human immunodeficiency virus transmission in Kenya and Zimbabwe. $J$ Infect Dis 1996;174(Suppl 1):S240-7.

16 Wong ML, Chan KW, Koh D. A sustainable behavioral intervention to increase condom use and reduce gonorrhea among sex workers in Singapore: a 2-year follow-up. Prev Med 1998;27:891-900.

17 Behets FM-TF, Andriamiadana J, Randrianasolo D, et al. Laboratory diagnosis of sexually transmitted infections in women with genital discharge in Madagascar: implications for primary care. Int J STD AIDS 2002;13:606-11.

18 Leutscher PDC, Behets F, Rousset D, et al. Sexual behavior and sexually transmitted infections in men living in rural Madagascar: implications for HIV transmission. Sex Transm Dis 2003;30:262-5.

19 Maynard-Tucker G. Etude qualitative des comportements sexuels es groupes a risque: prostituees non-fichees et clients pour la prevention du SIDA a Antananarivo, Madagascar. Projet Banque Mondiale CR 1967-MAG, The Futures Group International, 1996.

20 Behets FM-T, Andriamahenina R, Andriamiadana J, et al. High syphilis and low but rising HIV seroprevalence rates in Madagascar. Lancet 1996:347:831.

21 Behets F, Andriamiadana J, Rasamilalao D, et al. Sexually transmitted infections and associated socio-demographic and behavioral factors in women seeking primary care suggest Madagascar's vulnerability to rapid HIV spread. Trop Med Int Health $2001 ; 6: 202-11$.

22 Van Damme K, Rahamefy O, Behets F, et al. Structured, effective, and affordable counseling by peer educators to prevent STls among sex workers in Madagascar. Poster presented at the XIV International Conference on AIDS Volume 1. Barcelona, 7-12 July, 2002:249.

23 Fisher JD, Fisher WA. Theoretical approaches to individual-level change in HIV risk behavior. In: Peterson JL, DiClemente RJ, eds. Handbook of HIV prevention. New York: Kluwer Academic/Plenum Publishers, 2000.

24 Ford N, Koetsawang S. A pragmatic intervention to promote condom use by female sex workers in Thailand. Bull World Health Organ 1999:77:888-94.

25 Behets FMTF, Rasolofomanana JR, Van Damme K, et al. Evidence-based treatment guidelines for sexually transmitted infections developed with and for female sex workers. Trop Med Int Health 2003;8:251-8.

26 Steen R, Dallabetta G. Sexually transmitted infection control with sex workers: regular screening and presumptive treatment augment efforts to reduce risk and vulnerability. Reprod Health Matters 2003;1 1:74-90

27 Davies SC, Otto B, Partohudoyo S, et al. Sexually transmitted infections among female sex workers in Kupang, Indonesia: searching for a screening algorithm to detect cervical gonococcal and chlamydial infections. Sex Transm Dis 2003;30:671-9

28 Geary CW, Tchupo J-P, Johnson L, et al. Respondent perspectives on selfreport measures of condom use. AIDS Educ Prevent 2003;15:499-515.

29 Fishbein M, Pequegnat W. Evaluating AIDS prevention interventions using behavioral and biologic outcome measures. Sex Transm Dis 2000;27:101-10

30 Gollub E, French $P$, Latka $M$, et al. Achieving safer sex with choice: studying a women's sexual risk reduction hierarchy in an STD clinic. J Women's Health \& Gender-based Med 2001;10:771-83.

31 Kalichman S, Williams E, Nachimson D. Brief behavioural skills building intervention for female controlled methods of STD-HIV prevention: outcomes of a randomized clinical field trial. Int J STD AIDS 1999:174-81.

32 Artz L, Macaluso M, Brill I, et al. Effectiveness of an intervention promoting the female condom to patients at sexually transmitted disease clinics. Am J Public Health 2000;90:237-44.

33 Elwy AR, Hart GJ, Hawkes S, et al. Effectiveness of interventions to prevent sexually transmitted infections and human immunodeficiency virus in heterosexual men. Arch Intern Med 2002;162:1818-30.

34 Sanchez J, Campos PE, Coutois B, et al. Prevention of sexually transmitted diseases (STDs) in female sex workers: Prospective evaluation of condom promotion and strengthened STD services. Sex Transm Dis 2003;30:273-9.

35 Hearst N, Chen S. Condom promotion for AIDS prevention in the developing world: Is it working? Stud Fam Plann 2004;35:39-47. 